\title{
« Héros cellulaires » : arts, histologie et pédagogie
}

\section{"Cellular heroes": arts, histology and pedagogy}

\author{
Jean-François Bodart ${ }^{1,4}$, Adèle Tilouine ${ }^{1,2}$, Camille Vandromme ${ }^{1,2}$, Christine Vandromme ${ }^{3}$ \\ et Corentin Spriet ${ }^{1,2}$
}

\author{
${ }^{1}$ Univ. Lille, CNRS, UMR 8576-UGSF-Unité de Glycobiologie Structurale et Fonctionnelle, F-59000 Lille, France; \\ ${ }^{2}$ Univ. Lille, CNRS, Inserm, CHU Lille, Institut Pasteur de Lille, US 41-UMS 2014-PLBS, F-59000 Lille, France; \\ ${ }^{3}$ Lalo-Clément school, 3 Rue des Déportés, 59000 Lille. \\ ${ }^{4}$ Auteur correspondant, jean-francois.bodart@univ-lille.fr
}

RÉSUMÉ. « Héros cellulaires 》 est un projet inter-degré multidisciplinaire qui a associé 240 élèves, des professeurs du Grand Lycée Franco-Libanais de Beyrouth, ainsi que quatre experts extérieurs, scientifiques et/ou artistes de I'Université de Lille et du CNRS. À l'aide des informations que les chercheurs et les enseignants leur ont apportées, les élèves ont découvert que les cellules s'organisent en tissus pour accomplir des fonctions. Ils ont détourné les photographies des coupes de tissus d'animaux pour faire émerger des personnages imaginaires. L'analyse technique des matériaux a permis d'imaginer des personnages aux dimensions mythologiques, à partir des connaissances que les élèves avaient acquises au sujet des cellules observées. Chacun de ces héros a fait l'objet d'une description plurilingue (français, arabe, anglais), à partir des caractéristiques propres à l'élément microscopique sélectionné.

" Héros cellulaires » a permis aux élèves de prendre conscience de l'importance des démarches scientifiques, artistiques, et de la créativité qui constituent des compétences fondamentales à acquérir durant leur parcours individuel. Sont présentés ici des éléments de contexte concernant des approches artistiques et pédagogiques employées en relation avec l'histologie, ainsi que la méthodologie utilisée durant ce projet. "Héros cellulaires " a été valorisé par une exposition, la création d'un musée virtuel et a bénéficié d'un fort relais institutionnel par la mission laïque française et l'Agence pour l'enseignement français à l'étranger (aefe) Proche-Orient.

ABSTRACT. "Cellular Heroes " is an interdisciplinary project that involved 240 schoolchildren as well as teachers from the Grand Lycée Franco-Libanais of Beyrouth, and four external experts, scientists and / or artists from the University of Lille and the CNRS. Using information from researchers and teachers, schoolchildren discovered that cells organize themselves into tissues to perform functions. Based on their observations and the photographs of animal tissue sections, they were called to describe imaginary characters. The technical analysis of the materials enabled them to imagine characters with mythological dimensions, based on the knowledge that the schoolchildren had acquired about the cells observed. Each of these heroes was the subject of a multilingual description (French, Arabic, English), based on the characteristics specific to the selected microscopic elements.

"Cellular Heroes » engaged schoolchildren to become aware of the importance of the recognition of scientific methods, artistic and creativity approaches which are to be placed among the fundamental skills to be acquired during their individual career. Here are Contextual elements presented and discussed main elements regarding artistic and pedagogical approaches employed in relation to histology, as well as the methodology used during this project. The "cellular heroes" were promoted by an exhibition, the creation of a virtual gallery, and benefited from strong institutional support from the "mission laïque française" and the Middle East " Agence pour l'enseignement français à l'étranger". MOTS-CLÉS. histologie, paréidolie, pédagogie, créativité, tissu cellulaire, interdisciplinaire, atelier.

KEYWORDS. histology, pareidolia, pedagogy, creativity, cellular tissue, interdisciplinary, workshop.

\section{Présentation du contexte du projet "Héros Cellulaires"}

\subsection{Préambule}

Nous présentons ici une expérience interdisciplinaire, qui a mené à la production de "Héros cellulaires" par les élèves du Grand Lycée Franco-Libanais de Beyrouth. Cette expérience s'est appuyée sur une approche pédagogique et une étroite collaboration artistique et scientifique.

Au cours de ce projet, 240 élèves de sixième ont exécuté des productions artistiques à partir d'images de microscopie réalisées alors qu'ils étaient au CM2. À l'aide des informations que les chercheurs leurs ont apportées, les élèves ont détourné ces images scientifiques en y ajoutant des 
collages, dessins et peinture pour faire émerger des personnages imaginaires. Chacun de ces héros a fait l'objet d'une description en trois langues (français, arabe, anglais), à partir des caractéristiques propres à l'élément microscopique sélectionné, des suggestions des chercheurs et de l'imaginaire des élèves.

Cette expérience s'est enracinée dans une genèse, le projet "mythologie cellulaire" ${ }^{1}$, et a nécessité une préparation réalisée au Grand Lycée Franco-Libanais quant au choix et à l'organisation du matériel photographique et la constitution d'un référentiel culturel approprié. La réalisation proprement dite des œuvres s'est faite lors d'ateliers où les élèves ont pu bénéficier d'un accompagnement artistique, scientifique et linguistique. C'est une invitation à rêver la science, à laisser l'imagination envahir le domaine du scientifique, où le développement des technologies d'observation à l'échelle cellulaire (histologie et microscopie optique) permet de produire des corpus de matériaux avec des caractéristiques esthétiques.

Ce texte présente le contexte du projet, les éléments de réflexions pédagogiques qui ont présidé à la constitution des ateliers, ainsi que le déroulement de ces ateliers.

\subsection{Contexte de l'utilisation de I'histologie dans le domaine de l'art}

L'histologie et la microscopie sont des disciplines scientifiques propres à susciter l'émotion, qui mettent face à la beauté. La culture des sciences morphologiques «s'accorde (...) avec ce goût inné que nous avons pour la beauté des formes vivantes, depuis les plus élevées en organisation jusqu'aux plus humbles, avec cette recherche ardente de la vérité expressive et exprimable qui fournit à nos artistes des thèmes inépuisables et qui ajoute aux satisfactions intellectuelles du naturaliste quelques-unes des émotions de l'artiste. ${ }^{2}$

\subsubsection{Histologie}

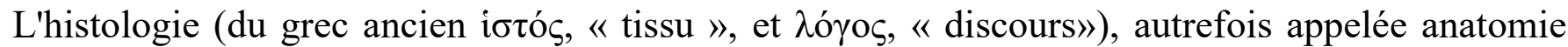
microscopique, est la branche de la biologie et de la médecine qui étudie les tissus biologiques. Marcello Malpighi est considéré comme un des fondateurs de l'histologie au XVIIème siècle : il corréla l'analyse des tissus vivants aux phénomènes physiopathologiques ${ }^{3}$. L'évolution et le développement de cette discipline se sont opérés au milieu du XIXe siècle au travers (1) du développement de la théorie cellulaire de Schleiden et Schwanen (1837), et (2) du développement du microscope : fort d'une technique alors vieille de deux siècles, le microscope optique est devenue achromatique et a permis la quantification fine de différentes couleurs à l'échelle du micromètre. Étroitement associée aux méthodes chimiques de coloration topographiques ou histochimiques, l'histologie révèle respectivement l'architecture tissulaire ou les actions conjuguées des colorants avec des molécules spécifiques. L'évolution de la discipline a ensuite été marquée par deux autres révolutions que constituent l'apparition de la microscopie électronique et la biologie moléculaire.

\footnotetext{
1 Mythologie Cellulaire (https://adeletilouine.wixsite.com/mythologiecellulaire) est un projet art-science qui vise à forger un panthéon de créature mythologiques cachées au sein de photographies de coupes histologiques au travers de visuels et de textes. Ce projet résulte d'une résidence artistique au laboratoire TISBio de juillet à août 2018 et a été développé en collaboration entre Adèle Tilouine, Jean-François Bodart et Corentin Spriet. II a donné lieu à deux expositions : de novembre 2018 à janvier 2019 à LILLIAD Learning Innovation Center, Villeneuve-d'Ascq et de mars à mai 2019 à la Bibliothèque Universitaire des Sciences Humaines et Sociales de l'Université de Lille, Villeneuve-d'Ascq.

${ }^{2}$ Leçon inaugurale par le Professeur COLLIN (1920) L'Histologie et la Médecine (chaire d'histologie de Nancy)

3 Marcello Malpighi (1628-1694). Fughelli P, Stella A, Sterpetti AV. Circ Res. 2019 May 10;124(10):1430-1432. doi: 10.1161/CIRCRESAHA.119.314936.
} 
Les ultrastructures tissulaires d'une échelle inférieure au micromètres sont devenues accessibles tandis que la biologie moléculaire ouvrait des perspectives d'analyses fonctionnelles, dépassant le cadre des études purement morphologiques.

Pour être étudiés en microscopie optique, les tissus biologiques sont chimiquement figés dans un état qui conserve les tissus dans une structure la plus proche possible de leur état physiologique. Cette étape, dite de fixation des tissus, vise à empêcher toute dégradation ou altération des tissus. Afin d'être analysés sous forme de fines coupes, les tissus sont ensuite préparés de manière à obtenir une consistance solide. A cette fin, les tissus subissent une étape d'inclusion dans une résine ou dans la paraffine qui s'infiltre et enrobe les tissus. Enfin, pour révéler leur architecture ou la présence de molécules spécifiques (acides nucléiques, lipides, collagène, polysaccharides), les coupes subissent une étape ou plusieurs étapes de coloration. La palette de ces colorations comprend des couleurs rouge, orange, vert, jaune, violet, bleu et noir qui s'associent dans des colorations dichromatiques (i.e. hématoxyline, colorant nucléaire / éosine, colorant cytoplasmique) ou trichromes. Par exemple, le trichrome de Gomori permet de distinguer les fibres collagènes dans le tissu conjonctif et fait apparaître des colorations noires, rouges, vertes ou bleues. D'autre colorations reposent sur les affinités des structures avec l'argent ou le chrome.

\subsubsection{Dimensions esthétiques de l'histologie}

Puisque l'histologie et la microscopie, qui mobilisent des techniques de recherche scientifique, jouent un rôle clé dans la révélation de la beauté de la nature, il y a eu une tendance à les considérer comme une forme d'art. Néanmoins, l'utilisation de l'histologie et de la microscopie en science pour corréler les structures cellulaires avec le fonctionnement des organes ou des organismes, se distingue fondamentalement de la créativité artistique qui conduit à la production de formes ou de représentations qui ne sont pas limitées par leur stricte adhésion à la réalité. L'approche scientifique implique une pensée critique ainsi qu'une objectivité scrupuleuse dans la relation avec le matériel biologique examiné ${ }^{4}$. Cette démarche tend à réduire toute transformation de l'objet observé, voire à récuser toute transformation.

Avant d'être photographiée, la coupe histologique était observée et dessinée mais, Marcello Malpighi (1628-1644) ou Antoni van Leeuwenhoek (1632-1723, considéré comme le père de la microscopie optique) n'ont jamais envisagé leurs dessins, aux qualités esthétiques exceptionnelles, comme des œuvres d'art. Aucune biographie de scientifiques dont les travaux étaient richement illustrés ou dont la microscopie constituait le cœur des travaux, ne réfère à ces scientifiques en tant qu'artistes. Ainsi la qualité du dessin ou de la photographie n'a pas présidé à une revendication du statut d'artiste pour les scientifiques concernés. Santiago Ramón y Cajal (1852-1934) peut faire figure d'exception ${ }^{5}$. Se destinant en premier lieu à une carrière artistique, Cajal conserva un goût prononcé pour le dessin, la peinture, le sens du détail et de l'harmonie esthétique sur lesquels il fondera son activité scientifique, qui révéla les interrelations entre les divers types de cellules du système nerveux ${ }^{6}$. Dans ses publications scientifiques, où la microscopie et des techniques de coloration tiennent des places centrales, Cajal utilise à la fois la peinture et la photographie comme supports au travers desquels il exprime sa créativité.

\footnotetext{
${ }^{4}$ Avramouli A, Gonidi M, Lambropoulou M. Microscopy as a form of art. Technoetic Arts: A Journal of Speculative Research Volume 15 Number 2

5 Santiago Ramón y Cajal - Neuroanatom, Künstler und Literat. Steininger H, Codina-Canet C. Dtsch Med Wochenschr. 2018 Dec;143(25):1847-1851. doi: 10.1055/a-0640-4652. Epub 2018 Dec 18.

${ }^{6}$ Cajal and the discovery of a new artistic world: the neuronal forest. DeFelipe J. Prog Brain Res. 2013;203:201-20.
} 


\subsubsection{Histologie : détournement sémantique}

On peut noter des appropriations, probablement anecdotiques mais révélatrices, du terme «histologie » : ce détournement élargit le champ sémantique du mot histologie. Le terme de coupes histologiques a pu être utilisé pour faire référence à des échantillonnages transversaux lors d'expositions ${ }^{7}$. Jean-François Jaeger, dans Psycho-sites, utilise la formulation d' «histologie de couches secrètes ${ }^{8}$. Cécile Reims mentionne quant à elle « une histologie de l'âme ${ }^{9}$. Le terme d'histologie renvoie dans l'imaginaire de plusieurs artistes au secret, à l'interne, à ce qui se dérobe au regard, ce qui est caché et nécessite une technologie particulière pour être révélé. Il est associé à l'idée de couches et de surfaces superposées.

\subsubsection{Approches artistiques impliquant l'histologie}

Plusieurs approches sont observées où (1) l'histologie constitue une source d'inspiration, (2) la coupe histologique fait partie intégrante de l'œuvre, ou (3) l'artiste manipule l'histologie. Ces démarches arts sciences se distinguent des œuvres qui utilisent la matière biologique vivante : transformation et altération de cette matière, création d'animaux transgéniques ou de végétaux incluant un gène humain ${ }^{10,11,12}$. En histologie, le matériel biologique qui est utilisé est inerte et n'est plus vivant.

L'histologie en tant que science peut être une source d'inspiration pour la création d'une œuvre. La coupe d'un tissu peut aussi être source d'inspiration, intégrée en tant que telle à l'œuvre ou transformée.

Quand la coupe ou la photographie de la coupe ne sont pas utilisées comme support physique de l'œuvre, le travail artistique se focalise sur des formes, des couleurs, des textures et des compositions inspirées par l'organisation des structures cellulaires comme dans les œuvres de Tolis Tatolas $^{13}$ (travail sur la géométrie et des abstractions) ou les gravures de Cécile Reims, où l'histologie, l'anatomie et la botanique sont des sources d'inspiration déclarées et revendiquées par l'artiste ${ }^{14}$.

La coupe histologique peut même être créée pour exister dans une dimension scientifique : un tissu imaginaire est produit dans une réalisation où l'histologie et l'embryologie apparaissent comme

7 https://www.fondationzervos.com/actualites/Docs/catalogue-expo-corpssproc.pdf catalogue électronique de l'exposition CorpssproC, Annick \& Louis Doucet, 2015.

8 J.F. Jaeger, dossier de presse. Matière et Mémoire : la Demeure du Patriarche - Dossier de presse https://issuu.com/agencecommunicart/docs/ib dp matiere memoire $2410 \mathrm{fb}$

${ }^{9}$ Cécile Reims, Bagages perdus. 2018 Editeur : le temps qu'il fait

${ }^{10}$ Catherine Voison, "L'art contemporain au prisme des biotechnologies », Amnis, 13 (2014)

${ }^{11}$ Eduardo Kac, Histoire Naturelle de l'Énigme (août 2003-2008). Pétunia exprimant un gène de l'artiste, A été réalisée grâce au soutien du Pr. Neil Olszewski du Collège des sciences biologiques et du département de biologie végétale de l'Université du Minnesota, St. Paul.

12 Laura Cinti, Howard Bolland, The Cactus Project C-Lab, Londres, 2009. Réalisation de cactus exprimant de la kératine (cheveu).

${ }^{13}$ Cell layers (2010) - Tolis Tatolas

14 Cécile Reims -« Les états du corps" / "L'oeuvre gravée 1945-2011" 
des démarches nécessaires à l'étude d'une espèce. Ainsi la démarche de la plasticienne Ema Eygreteau inclut des dessins et peintures histologiques, notamment dans l'œuvre Gossipium 3.0 avec la mise en culture d'une cellule mi-végétale mi-humaine ${ }^{15}$.

La coupe en tant qu'objet (lame, lamelle, tissu) et le microscope peuvent faire physiquement parties intégrantes de l'œuvre, en sus des photographies des coupes: Aurélie Mourier dans son exposition «approche discrète », a utilisé des lames, des microscopes et des photographies de coupes d'embryons de roussette ${ }^{16}$.

Ensuite, les tissus biologiques (conservés, colorés et présentés selon les techniques d'histologie) peuvent être présentés en tant qu'œuvres ou constituer le support de l'œuvre, modifiés par un travail plastique. Des images d'histologie (thyroïde, intestin, foie, os, pancréas, œsophage, testicule, rein) ont été utilisées pour la création de céramiques par Emily Evans ${ }^{17}$ : les coupes histologiques avaient été fournies par Michelle Spear, anatomopathologiste de l'Université de Cambridge. En juillet 2012, Véronique Chobaz et Nathalie Busso, de formations scientifiques, ont proposé une exposition de photographies de tissus d'articulations où les photographies étaient présentées sans travail plastique, sous forme brute ${ }^{18}$. Professeur au laboratoire d'histologie et d'embryologie dans le département médical de la Democritus University of Thrace (Grèce), Maria Lambropoulou expose régulièrement des photographies d'histologie où les formes et structures exposées montrent des ressemblances avec des objets de l'imaginaire collectif (soulier de Cendrillon), des papillons ou des coeurs ${ }^{19}$. Si le qualificatif d'œuvres pour ces photographies est discutable ${ }^{20}$, ces expositions d'images microscopiques visent à stimuler l'imagination, créer une esthétique agréable ou une réponse émotionnelle positive.

Notre démarche, à l'instar de celle d'Eric Philippe et de Guy Lemieux, ${ }^{21}$ s'inscrit dans une collaboration plus bilatérale entre scientifiques et artistes. Éric Philippe, professeur au Département de chirurgie, division anatomie de la Faculté de médecine de l'université de Laval, et Guy Lemieux, artiste peintre, ont étroitement collaboré avec des étudiants qui n'étaient ni artistes ni scientifiques lors des écoles internationales d'été de Percé de l'Université Laval. Ces étudiants ont été confrontés à la fois aux techniques picturales et aux étapes de la formation du corps humain et des différents tissus histologiques qui le composent. Ces ateliers de création en arts visuels ont permis, de 2009 à 2012, de créer des œuvres traduisant les formes, les textures, les transparences et les couleurs des tissus du corps humain.

\footnotetext{
${ }^{15}$ Ema Eygreteau. Gossipium 4.0 - février-mars 2020, Bordeaux. Gossipium 3.0 BIENNALE ORGANO : 18 - 19 MAI 2019

${ }^{16}$ Aurélie Mourier, artiste en résidence artistique à Montpellier 2. Exposition Approche discrète, Privas, mai-juin 2015.

17 "eat your heart out" event at the St Batholomew's Hospital Pathology Museum, London October 2012. http://www.medinart.eu/works/emily-evans/

18 "Art-iculation" (2012, Romont) / prix à la création Zonta 2012.

19 Lambropoulou M, Pasadakis P, Papadopoulos. Tissue Love Symbols. International Journal of surgical Pathology (2015) ; http://www.medinart.eu/works/maria-lambropoulou
}

\footnotetext{
${ }^{20}$ Avramouli A, Gonidi M, Lambropoulou M. Microscopy as a form of art. Technoetic Arts: A Journal of Speculative Research Volume 15 Number 2

21 "Quand I'histologie devient une oeuvre d'art » - Eric Philippe - congrès de l'Acfas (Association francophone pour le savoir), 2013, Université de Laval
} 
Plusieurs écueils existent au sein des collaborations arts-sciences. D'une part, l'artiste peut être employé comme simple illustrateur ou faire-valoir par le scientifique. D'autre part, l'artiste peut commettre des erreurs d'interprétation et employer les données scientifiques, réalisant ainsi des productions qui engagent des controverses sans fondements scientifiques et contribuent à la dissémination de contenus scientifiques erronés. Il existe donc une forte dimension éthique du «faire ensemble» où les injonctions et valeurs implicites du travail artistique et du travail scientifique doivent être respectées.

Notre travail s'est inscrit dans une démarche, où ce n'est pas le matériel biologique qui est manipulé à proprement parler, mais sa représentation. Il ne s'agit pas de sortir des altérités biologiques depuis les coulisses de la science, mais de transformer et déplacer les représentations du tissu biologique par le biais de la créativité, et de proposer des altérités de représentations qui soient utilisées pour des fins pédagogiques ou simplement pour s'approprier la structure de son corps.

\section{Approche employée}

La pédagogie est un des angles principaux par lequel nous avons abordé ce projet arts \& sciences, dans l'objectif d'employer des méthodes qui permettent le développement et l'ancrage de nouvelles compétences, ainsi que l'explicitation de valeurs. Cette démarche propose d'aller au-delà de la «simple» démystification du corps et des tissus qui composent les organismes vivants, afin de permettre aux élèves de s'approprier de nouvelles représentations des tissus biologiques. Notre méthode d'appropriation s'est largement appuyée sur le phénomène de paréidolie. Ce phénomène est une forme d'illusion d'optique où le cerveau donne sens à un stimulus visuel indéfini et vague pour que nous l'interprétions comme une forme précise, le plus souvent animale ou humaine (voir 2.2).

\subsection{Considérations éthiques}

Notre travail sur la compréhension du fonctionnement des organes vivants et la manipulation des structures histologiques a été réalisé via un "matériel" inerte, figé, qui ne pose aucun problème éthique lié à la "manipulation" du vivant. Ainsi le bien-fondé de ces expérimentations artistiques sur un matériau biologique photographié est moins questionné, sur le plan éthique, par une société soucieuse de sa survie au sein d'un environnement, dont elle prend conscience de la vulnérabilité.

\subsection{Paréidolie et images scientifiques}

La paréidolie est un phénomène ou biais cognitif relié à la capacité du cerveau à structurer en permanence son environnement, à transformer les informations fournies par la rétine ou par l'oreille en objets connus, et donc à donner un sens, à extraire une forme d'un matériau aléatoire. Le phénomène de paréidolie apparaît très tôt chez l'enfant, dès l'âge de 10 mois ${ }^{22}$. La paréidolie faciale est la plus explorée, qui tend à voir des visages dans les nuages ${ }^{23}$ ou observer des figures religieuses dans la nourriture ${ }^{24}$. Le phénomène de paréidolie n'est pas limité aux stimuli visuels, et explique également la tendance à dégager un message sonore lorsqu'une bande son est passée à l'envers. La paréidolie faciale est idéale pour comprendre comment le cerveau intègre des données et effectue

\footnotetext{
22 Kato M, Mugitani R (2015) Pareidolia in Infants. PLoS ONE 10(2): e0118539. doi:10.1371/ journal.pone.0118539

${ }^{23}$ Guthrie S. Faces in the clouds. Oxford: Oxford University Press; 2015

24 Liu J, Li J, Feng L, Li L, Tian J, Lee K. Seeing Jesus in toast: neural and behavioral correlates of face pareidolia. Cortex. 2014;53:60-77.
} 
une « reconnaissance faciale ${ }^{25}$, et tient une place de choix dans les processus de création artistique $^{26}$, en dépit de sa qualité de biais dans la perception, puisque la paréidolie est une illusion.

La paréidolie est également utilisée à des fins pédagogiques dans différents domaines comme ceux de la médecine et de la radiologie. Elle constitue une des stratégies pour aider les étudiants et doctorants à appréhender et interpréter des images médicales complexes, afin de reconnaître des tableaux cliniques. Parmi les images couramment utilisées, on peut noter : le terrier écossais, la chouette qui cligne de l'oeil, l'étoile de mer, l'oiseau guêpier, le serpent prêt à mordre ${ }^{27}$. Les animaux peuvent être reconnus par le biais de le paréidolie dans les images produites par neuroimagerie, en fonction de la présence de tableaux cliniques spécifiques. En associant un aspect radiologique donné à la morphologie d'un animal (le panda et la maladie de Wilson, l'éléphant et la maladie d'Alzheimer, les papillons et les glioblastomes), les étudiants comme les praticiens améliorent leurs compétences diagnostiques et renforcent des stratégies mnémotechniques dans la pratique de la radiologie ${ }^{28}$.

L'utilisation de structures qui montrent des ressemblances avec des formes habituellement observées dans l'environnement quotidien ${ }^{29}$, dans le cadre de cours d'enseignement aux étudiants en médecine, facilite la compréhension des structures microscopiques plus facile et augmente l'attractivité de ce domaine scientifique considéré parfois comme austère ${ }^{30}$. L'emploi d'images histologiques ressemblant à des œuvres d'art est également considéré comme un outil de choix pour enseigner l'histologie ${ }^{31}$ : ces images permettent d'enseigner les structures physiologiques normales, en intégrant des informations culturelles supplémentaires (i.e. le cri, d'Edvard Munch, ou le Pèlerinage à la source Saint-Isidore, de Francisco Goya). L'enseignement est rendu plus interactif et intéressant.

Les mécanismes cognitifs mis en œuvre par la paréidolie ont été utilisés sur deux versants durant notre démarche (figure 1.A) :

- Stimuler la créativité des élèves en les invitant à exprimer les formes reconnues dans les tissus, même si ces formes ne sont pas corrélées aux caractéristiques histologiques ou fonctionnelles du tissu (exemple : un aspect granuleux rappelant les grains de riz a conduit des élèves à inventer un hommesushi).

\footnotetext{
${ }^{25}$ Barik K, Daimi SN, Jones R, Bhattacharya J, Saha G. A machine learning approach to predict perceptual decisions: an insight into face pareidolia. Brain Inform. 2019 Feb 5;6(1):2.
}

${ }^{26}$ Lee Joanne. I See Faces : popular pareidolia and the proliferation of meaning. In A. Malinowska and K. Lebek, Materiality and popular culture : the popular life of things. Routledge. 2016

${ }^{27}$ Fatehi D, Salehi M. G, Farshchian N, Farzizadeh M, Mohammadi M, Rostamzadeh A. Pareidolia As Additional Approach To Improving Education and Learning In Neuroradiology; New Cases And Literature Review. Biomed Pharmacol J 2016;9(1)

${ }^{28}$ Maranhão-Filho, P., \& Vincent, M. B. (2009). Neuropareidolia: Diagnostic clues apropos of visual illusions. Arquivos de NeuroPsiquiatria, 67(4), 1117-1123.

${ }^{29}$ Lambropoulou M, Pasadakis P, Papadopoulos. Tissue Love Symbols. International Journal of surgical Pathology (2015) ; http://www.medinart.eu/works/maria-lambropoulou

\footnotetext{
${ }^{30}$ Avramouli A, Gonidi M, Lambropoulou M. Microscopy as a form of art. Technoetic Arts: A Journal of Speculative Research Volume 15 Number 2

${ }^{31}$ Art and Human Expression in Histology Ariane Ruyffelaert, Ingrid Garzón, José Aneiros-Fernández and Víctor Carriel INT J SURG PATHOL 2014 22: 525
} 
- Accompagner les élèves à la reconnaissance de formes dans les tissus (exemples : identification des formes flagellées des spermatozoïdes ou des différentes couches dans une coupe de peau). La paréidolie permet de créer des repères topographiques, puis d'enrichir l'ensemble perceptuel de l'élève. Cet enrichissement facilitera la mémorisation et la reconnaissance ultérieure de structures.

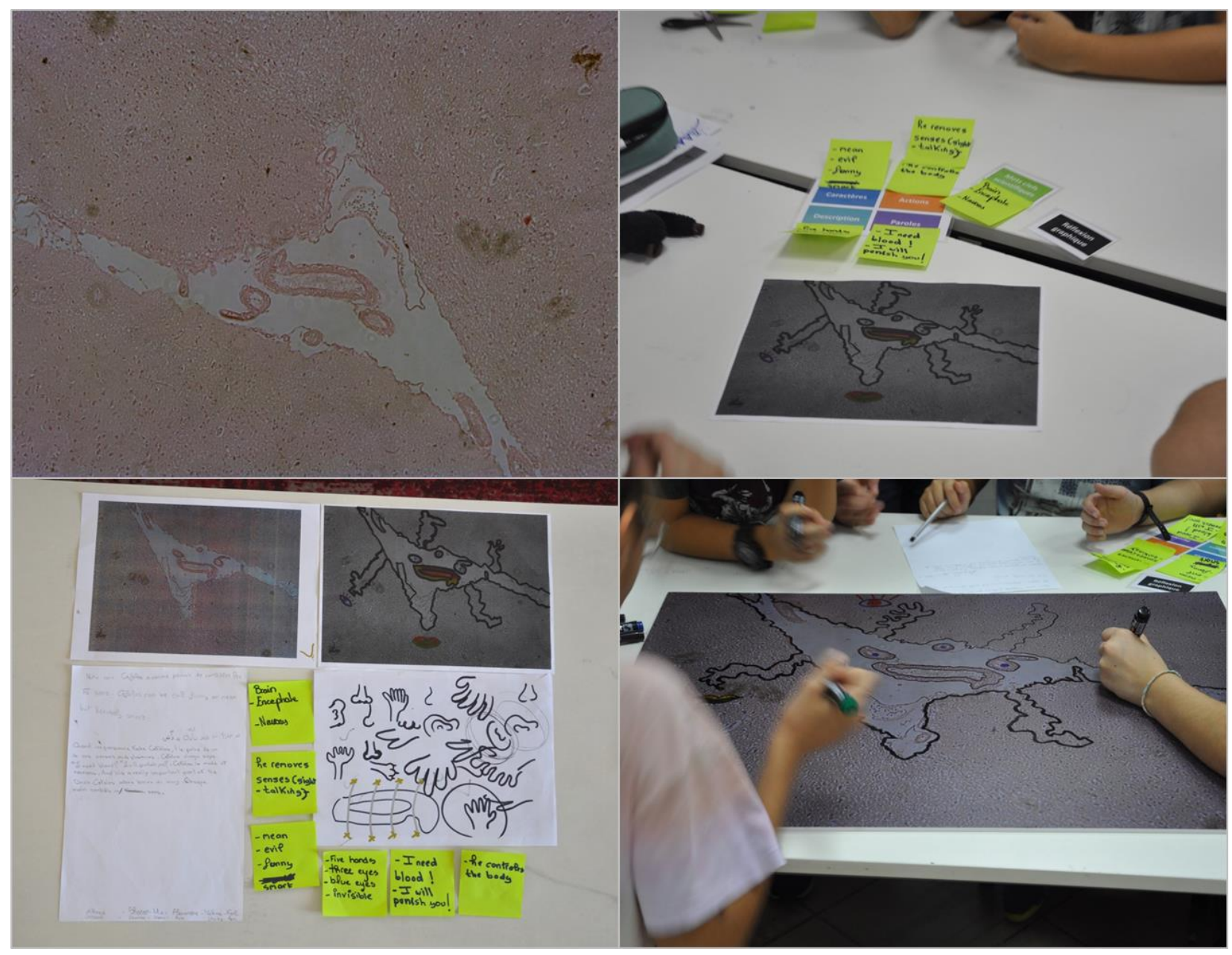

Figure 1. Illustration du processus créatif mis en œuvre dans le cadre des héros cellulaires. (A) Les élèves commencent par sélectionner une image issue d'un tissu cellulaire, ici, une coupe d'encéphale (grossissement 40X), puis (B) ils doivent définir ensemble les mots clefs de leur héros en répartissant des caractéristiques entre "caractère ", " description ", "paroles ", "actions » et " mot clef scientifique ». À partir de ce travail préparatoire, ils vont réaliser différents tests $(C)$, graphiques et écrits avant de passer à la réalisation de l'œuvre elle-même $(D)$.

\subsection{Compétences et valeurs véhiculées par l'atelier}

\subsubsection{Compétences}

Les compétences considérées et travaillées ont été les suivantes : (i) s'approprier des outils et des méthodes : utiliser le matériel adapté pour mener à bien une observation, (ii) utiliser des outils numériques : garder une trace numérique des observations (microscope et caméra numérique), (iii) comprendre, s'exprimer en utilisant le français, l'arabe, l'anglais à l'oral et à l'écrit, (iv) comprendre et s'exprimer en utilisant le langage scientifique, (v) décrire et interroger à l'aide d'un vocabulaire spécifique ses productions plastiques, (vi) justifier des choix pour rendre compte du cheminement qui conduit de l'intention à la réalisation, (vii) formuler une expression juste de ses émotions en prenant appui sur ses propres réalisations plastiques, celles des autres élèves et des œuvres d'art. L'ensemble de ces compétences matérialise que ce travail mené en inter-degré (CM2 - sixième) est réalisé de manière interdisciplinaire : Enseignement Intégré de Science et Technologie (EIST), Arts 
plastiques, Langues. Il a également permis aux élèves de découvrir un monde professionnel, en décloisonnant les milieux artistiques et scientifiques.

\subsubsection{Valeurs}

Dans une attitude réflexive et par souci de déontologie, les animateurs ont souligné les valeurs de coopération, de créativité, de rigueur scientifique et de pédagogie, en montrant comment ces valeurs avaient pu être mises à l'œuvre dans le projet mythologie cellulaire et pouvaient être employées dans ce projet. L'encadrement pédagogique a pris soin de rendre explicite les valeurs mobilisées ${ }^{32}$. Une valeur est un critère, une référence, un idéal qui permet d'apprécier et d'évaluer notamment des actes et des situations ${ }^{33}$ : «toute valeur, en effet, nous donne un conseil et nous suggère une orientation, puisqu'elle nous indique dans quel sens nous pourrions souhaiter que le monde évolue ou dans un état où nous souhaitons qu'il se maintienne»» ${ }^{34}$.

Le travail a été réalisé par groupe de 5 élèves, chaque groupe d'élèves réalisant une production. La coopération entre élèves est donc une valeur qui a été soigneusement mise en avant. Elle constitue une action de «prendre part, concourir à une œuvre commune ; contribuer, participer à ». La coopération est une valeur que les enseignements doivent contribuer à promouvoir : la coopération est devenue un critère attendu dans la mise en œuvre de compétences (Ministère de l'éducation nationale et de la Jeunesse, $2018^{35}$ ). L'impact de la coopération entre élèves sur leurs performances scolaires est notable en sixième ${ }^{36}:$ «Il en ressort des progrès repérés chez tous les profils d'élèves, ainsi que des bénéfices en matière de solidarité mutuelle, d'autonomie et de responsabilité ». Dans le contexte géopolitique du Liban en novembre 2019, cette valeur du «faire ensemble » prenait implicitement une dimension d'autant plus critique.

Les enseignants mobilisés ont mis en relief le travail d'équipe, qui apprend à vivre et travailler ensemble dans le respect d'autrui. Les élèves ont ainsi été amenés à prendre conscience qu'ils travaillaient pour une œuvre commune, un ensemble qui bénéficierait à tous.

\section{Description et déroulement des ateliers}

\subsection{Comment stimuler la créativité des élèves?}

Pour accompagner et stimuler la créativité des élèves, une activité avec des cartes (réflexion graphique, mots clefs scientifiques, caractères, actions, paroles, description) et des post-it a été proposée.

En début d'atelier sont exposées sur la table les cartes «réflexion graphique » et «mots clefs scientifiques », de manière à ce que les élèves se remémorent les aspects caractéristiques et scientifiques du tissu qu'ils ont choisi. De la même manière, les élèves récapitulent ce qu'ils ont

\footnotetext{
32 Connac, S. (2017). La coopération entre élèves. Futuroscope : Réseau Canopé.

33 De la déontologie enseignante. Eirick Prairat Quadrige / PUF 2009.

34 P. Livet, Les normes, Paris, Armand Colin Coll. « vocation philosophique », 2006, p30.

${ }^{35}$ Ministère de l'éducation nationale et de la Jeunesse. (2018). Programme du cycle 3 : en vigueur à compter de la rentrée de l'année scolaire 2018-2019. http://cache.media.eduscol.education.fr/file/programmes_2018/20/2/Cycle_3_progra mme_consolide_1038202.pdf
}

${ }^{36}$ Sylvain Connac Dans Les Sciences de l'éducation - Pour l'Ère nouvelle 2018/4 (Vol. 51), pages 11 à 42. 
imaginé en regardant les coupes de tissus durant les cours d'arts plastiques. Le croquis réalisé en cours d'arts plastiques est posé à côté de la carte « réflexion graphique », pour les aider.

Ensuite les cartons plastifiés « caractères », « actions », «paroles », « description » sont posés au centre de la table et des post-it sont distribués. Les 4 catégories sont conçues pour stimuler l'imagination. Pour chaque élève, il est indiqué de prendre deux post-it et d'écrire sur les bouts de papier les mots clefs, en rapport avec le héros imaginé. La consigne suivante est donnée aux élèves : «Prenez deux à cinq minutes puis posez les post-it sur la table, et on discutera tous ensemble » (figure 1.B-C).

Si les élèves n'arrivent pas à trouver des mots clefs relatifs à leur personnage ou peinent à imaginer un personnage, leur imagination et les connaissances sont sollicitées en posant des questions : «comment est-il ? Qu'est-ce qu'il/elle aime ? Ou déteste ? Qu'est-ce qui t'inspire dans l'image de microscopie ? Est-ce qu'il y a un mot scientifique qui en évoque un autre ? Quel est le but dans la vie de ton héros ? Qu'est-ce qu'il veut réussir ?» L'animateur possède une carte supplémentaire : la carte «joker narrateur» offre à l'animateur l'opportunité de canaliser une histoire et de cadrer les idées qui s'éloignent trop des objets scientifiques observés. Dans le cas du projet «Héros cellulaires », les animateurs ont dû garder à l'esprit qu'une œuvre serait produite en parallèle par le collectif d'artistes et d'enseignants-chercheurs, en cohérence avec les quarante productions des élèves (figure 3.A).

\subsection{Déroulement}

Le déroulement de chaque atelier a été programmé de la manière indiquée dans le tableau 1 . Durant la séance, les animateurs et les encadrants ont insisté sur plusieurs points. En premier lieu le contexte du travail et de l'atelier ont été rappelés :

- «Le travail que nous allons faire ensemble est un travail avec de multiples dimensions. »

- «Ce que vous avez commencé à faire est exactement la démarche que les animateurs des ateliers ont suivie. A partir de photographies de microscopies, il a fallu laisser parler son inventivité et sa créativité. »

- «C'est aussi une démarche qui permet de faire le lien entre l'artiste et le scientifique, deux « figures » que l'on considère parfois comme étant très différentes. On associe l'artiste avec la créativité, l'ouverture d'esprit, l'originalité et la liberté ; on associe plutôt le scientifique avec la curiosité, l'esprit critique, l'expérimentation, et le raisonnement ou la rigueur. Mais par exemple, la créativité est très importante dans le travail scientifique. Les valeurs associées à l'art sont aussi importantes en sciences, et vice-versa. »

Le travail de préparation a été ensuite rappelé :

- «En CM2, vous avez travaillé et acquis des notions, du vocabulaire autour de la cellule. »

- «Nous allons nous appuyer sur ce que vous avez fait en cours d'arts plastiques, pour le graphisme. »

Enfin, nous nous sommes attardés sur les notions de compétences qui incluent les idées de dépassement, d'improvisation, de prise de risque, de prise de chemins de traverse ou de possibilité de commettre des erreurs. Les aspects coopératifs, le droit à l'erreur et à l'expérimentation ont été soulignés tout au long de la séance de travail :

- «Vous avez travaillé en groupe, sur un personnage, un héros. Nous allons continuer à travailler et à coopérer pour concrétiser ces personnages. » 
- «L'essentiel est de le faire ensemble, en respectant les émotions et la sensibilité de chacun, pour obtenir un résultat qui puisse nous plaire et que nous soyons heureux d'avoir produit. Nous allons le réaliser en parlant ensemble, en s'écoutant. »

- «Il ne faut pas avoir peur de tâtonner, d'expérimenter : on explore, on se trompe, on recommence, on réussit. »

Les consignes de dessin ont été les suivantes, avec des contraintes relatives au travail sur les photographies imprimées sur des plaques de PVC Expansé : réaliser en premier un dessin avec une mine fine, une mine plus grasse pouvant être utilisée au besoin par la suite (figure 1.D) ; les couleurs ne peuvent pas être éclaircies ; il est difficile de réaliser des aplats de couleur ; les plaques doivent être manipulées avec soin (rayures, propreté des mains propres); des essais peuvent être réalisés au dos des plaques de PVC, mais la manipulation des plaques doit être limitée et surveillée pour éviter d'abimer les photographies imprimées.

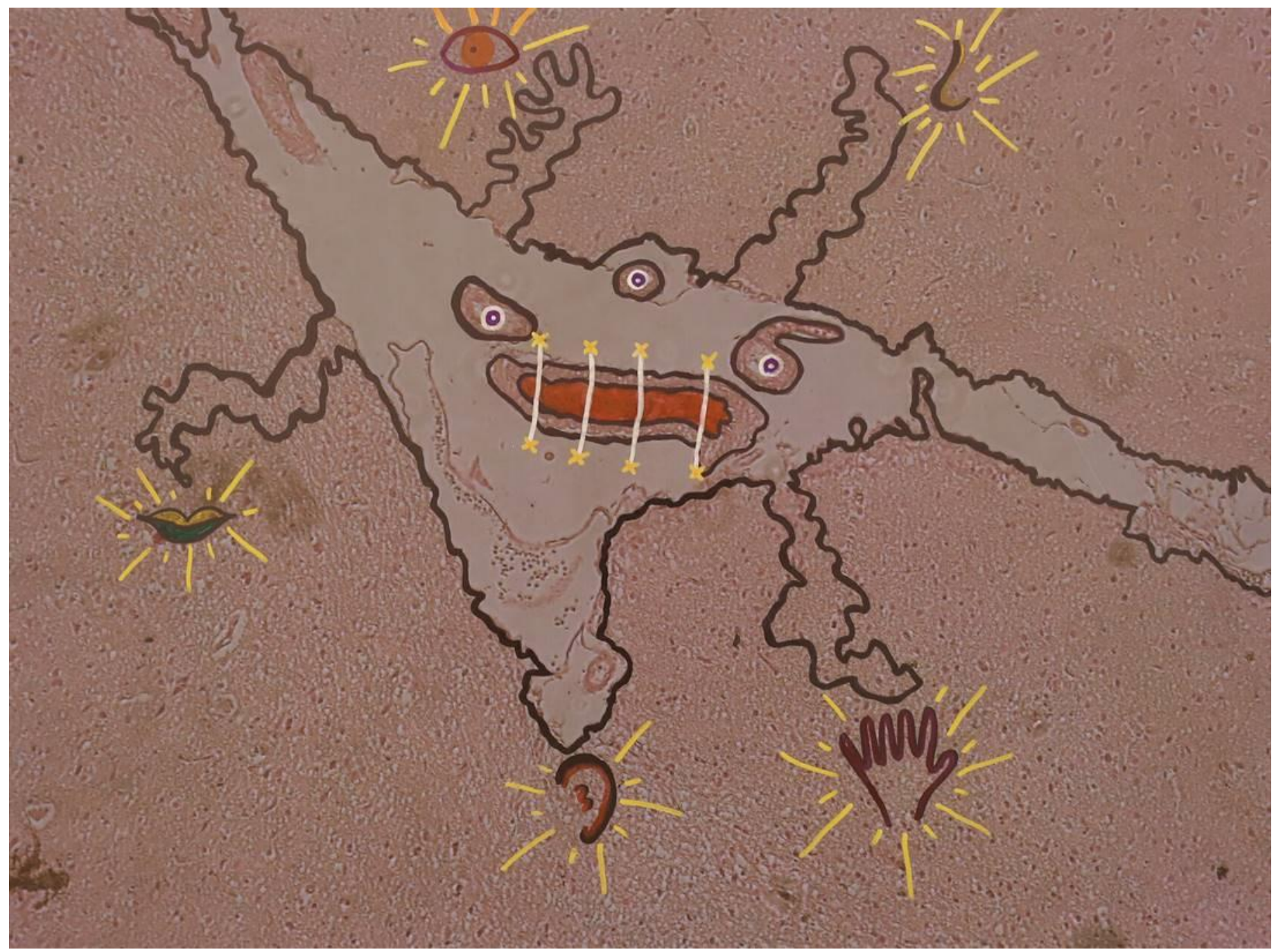

Figure 2. Résultat de la production décrite dans la figure 1 et réalisée par Karl Elie, Ahamad, Sharon, Nehme, Mia Maria. La description de ce héros, nommé Cefalos, est représentative des productions où les enfants ont préservé la cohérence entre le texte, la photographie et la nature du tissu biologique.

"Notre ami Cefalos a le pouvoir de contrôler les cinq sens. Cefalos can be evil, funny or mean but he's

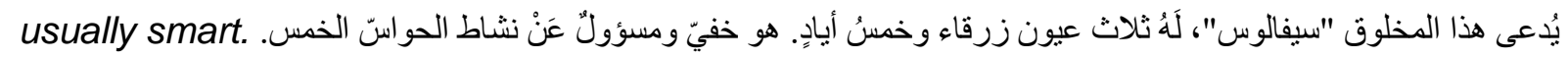
Quand une personne fâche Cefalos, il le prive de l'un de ses sens ou de plusieurs d'entre eux. Cefalos always says "I need blood! ", "I will punish you » Cefalos is made of neurons. And he is a really important part of the brain. Cefalos adore boire du sang. Chaque main contrôle un sens. ” 


\section{Retour d'expérience et valorisation}

\subsection{Productions des élèves}

Dans leurs productions, les élèves ont majoritairement intégré des fonctions qu'ils attribuent aux tissus (figure 2 et 3 B1-3). Sur les 40 productions réalisées, 39 font références aux tissus utilisés et 33 créations se construisent concrètement autour de la nature des échantillons biologiques. Lorsque le tissu proposé est une coupe de peau, ou d'épithélium glandulaire d'amphibien, la peau est un élément capital du héros décrit et la sensibilité est une caractéristique principale du héros : «I feel everything ». La protection est clairement une caractéristique principale du héros : «protective », « le protecteur », « il protège », « protect... and save... », « pieuvre protectrice ». Pour les coupes d'encéphale ou de cervelet (cellules nerveuses de Purkinje), les héros ou monstres imaginés montrent des qualités de sagesse, d'intelligence, ou des traits comme la nervosité. Ces héros peuvent être capables d'exercer une domination («il donne des ordres », « il est assis sur son trône »), par un contrôle des sens ou des émotions (" control of his enemy's feeling »). Quand les tissus biologiques observés sont des coupes de gonades (testicules), les héros sont des créateurs, ou des dieux de la fertilité, et génèrent la vie ( «baby maker », « canon à bébés », « déesse mère », « créer des enfants »), ou la protège («protect the children in distress »). Parfois, la narration ne fait pas explicitement référence à la fonction, mais nous avons noté que le nom du héros ou sa représentation graphique sont associés au serpent dans trois productions inspirées par les organes mâles de reproduction. Enfin, les coupes de rétine de bœuf ou d'œil de singe inspirent des héros possédant des super pouvoirs liés à la vision : les yeux sont plus grands ou émettent des rayons lasers. Le nombre des yeux du héros est un élément de narration : cyclopie (le « cycloptaure »), yeux surnuméraires ( des yeux partout sur le corps »; «10000 autres yeux »).

\subsection{Dimensions pratiques de l'atelier et de son déroulement}

Une durée de 3 heures a suffi aux élèves, à créer, et finaliser les dessins et textes demandés (en trois langues) en respectant les consignes. Certains élèves ont éprouvé des difficultés à comprendre les consignes des cartes « réflexions graphiques » et «parole »; de nouvelles formulations pourraient être testées (i.e. reformulation de la carte « réflexions graphiques » en carte «Que voyezvous? »). Une augmentation de la durée de l'atelier aurait accru le risque de détérioration des plaques de PVC. Pour autant, cette durée ne permet pas d'exploiter pleinement la paréidolie, pour que les élèves associent des formes avec les tissus, et utilisent ces formes comme moyens mnémotechniques de reconnaissance des tissus. Il serait nécessaire que les enseignants familiarisent plus les élèves avec la paréidolie. La conscience de ce phénomène inconscient pourrait amener les enseignants à renforcer la reconnaissance par les élèves des structures histologiques et les conduire à développer leurs propres exemples de paréidolie ou de repères topographiques pour faciliter la compréhension de la structure de la coupe.

Les élèves, à l'issue d'une phase réflexive même courte, reconnaissent rapidement la valeur du travail en groupe et verbalisent l'intérêt de cette coopération. Cette valeur a été explicitée et institutionnalisée. 

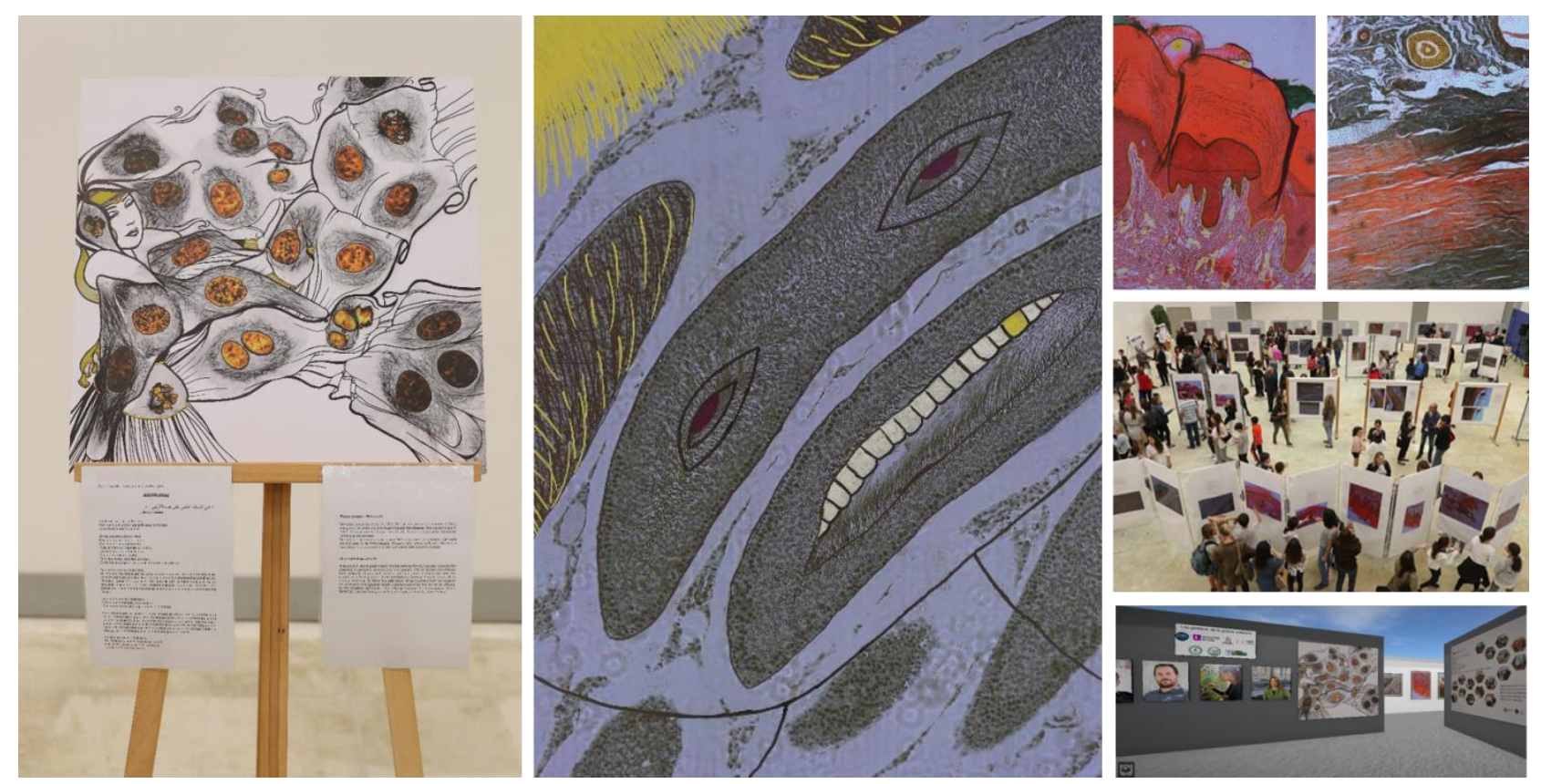

Figure 3. Vernissage de l'exposition au GLFL de Beyrouth. Production des animateurs (A), des élèves (B13). Lors du vernissage, les enfants ont eu l'occasion de présenter à la fois le concept de l'atelier mais également leur travail de groupes, renforçant l'acquisition des valeurs portées lors de l'atelier (C). (D) Une galerie virtuelle a également été mise en place afin de leur permettre de prolonger l'exercice chez eux et de garder un souvenir de l'évènement (https://www. artsteps.com/view/5e15eb1021487634d8881283).

\subsection{Valorisation et prospective}

Le vernissage de l'exposition des productions des élèves du Grand Lycée Franco-Libanais de Beyrouth a réuni plus de 800 personnes (figure 3C). Les élèves du GLFL ont réalisé un court reportage pour présenter ce travail inter-degrés ${ }^{37}$. Un musée virtuel a été créé pour que l'expérience se prolonge et puisse être partagée ${ }^{38}$.

L'ensemble de l'exposition et du musée virtuel (figure 3D) qui la complète ont offert une vitrine sur le projet pour les parents, l'ambassade, l'Agence pour l'enseignement français à l'étranger et la Mission Laïque. L'exposition a bénéficié d'un fort relais institutionnel grâce notamment aux sites de la mission laïque française ${ }^{39}$, de l'agence pour l'enseignement français à l'étranger du ProcheOrient ${ }^{40}$ et au site des reporters de l'agence pour l'enseignement français à l'étranger ${ }^{41}$. Un article relatant le projet «Héros cellulaires ${ }^{42}$ a figuré en première page du site de la mission laïque française parmi les 10 projets qui font l'actualité sur l'ensemble du réseau. «Héros cellulaires »a également fait la une des pages d'accueil de l'Agence pour l'enseignement français à l'étranger Proche-Orient.

\footnotetext{
${ }^{37}$ https://www.youtube.com/watch?v=Oa3Tf7amHHQ

${ }^{38}$ https://www.artsteps.com/view/5e15eb1021487634d8881283

39 http://www.mlfmonde.org/

${ }^{40}$ https://www.aefe-proche-orient.net/

${ }^{41}$ https://jriaefe.tumblr.com/

42 https://www.mlfmonde.org/un-travail-original-et-interdisciplinaire-au-glfl/
} 
Du point de vue des élèves, il est intéressant de constater qu'ils regardent le même tissu cellulaire mais ne voient pas les mêmes choses : ils peuvent s'attacher à la photo du tissu dans sa globalité ou se focaliser sur un détail, que ce détail soit constitué par un groupe de cellules, un artefact de coupe ou une cellule. Durant les phases d'échanges et de coopération, chaque enfant a le droit d'exprimer ce qu'il voit. Ainsi, les visions multiples, issues de profils cognitifs différents amènent le groupe à construire une représentation collective. Dans la mesure où le travail collectif est une condition pour l'école inclusive, cette perspective a amené le projet à se déployer dans une nouvelle variation, au sein de l'établissement Lalo-Clément de Lille. "Une aventure cellulaire pour une école inclusive et inventive » est organisée par un collectif d'enseignants, d'enseignants-chercheurs, de chercheurs, artistes et animateurs du temps périscolaire, pour l'ensemble des élèves de l'école, du CP au CM2, ainsi que des élèves d'une unité d'enseignement en élémentaire pour autistes (UEEA) et des élèves scolarisés en unité localisée pour l'inclusion scolaire (ULIS). L'équipe a engagé une profonde réflexion sur l'inclusion, s'appuyant sur les dimensions pédagogiques, scientifiques et artistiques des "Héros cellulaires ». Cette démarche, en appréhendant différentes perspectives de travail, permettra à la diversité des profils cognitifs de s'exprimer, et ainsi de renforcer l'acquisition de nouvelles compétences et aptitudes.

\section{Conclusion}

Cette expérience interdisciplinaire aura permis de développer la créativité des élèves, de favoriser leur confiance en soi, ainsi que de les amener à faire des liens entre arts et science. Ils ont pu découvrir la culture scientifique et ses exigences. L'atelier leur a offert de se confronter différemment à la réalité des tissus biologiques et de décentrer leur regard. D'un point de vue créativité, les élèves ont été confrontés à un exercice où les règles n'entravent pas, voire stimulent l'imagination et permettent d'appréhender un sentiment de liberté tel qu'il s'exerce dans les œuvres de Georges Perec ou du mouvement littéraire de l'Oulipo. Cette démarche augmente la sensibilité d'un jeune public aux démarches scientifiques et a permis au collectif constitué de contribuer au développement de nouvelles pratiques pédagogiques. La réussite de cette expérience interdisciplinaire nous engage à travailler la transposabilité d'un atelier arts-science où les élèves apprennent à être acteurs de leurs apprentissages et de la construction de leurs savoirs.

\begin{tabular}{|l|l|}
\hline Première heure & $\begin{array}{l}\text { Présentation du projet (10 minutes) } \\
\text { Les enfants verbalisent des mots clefs relatifs à leur travail de préparation et } \\
\text { mettent en relation le tissu choisi et le personnage imaginé. Utilisation } \\
\text { d'un« jeu de post-it » pour stimuler la prise de parole et l'expression (30 mi- } \\
\text { nutes). L'objectif au terme des } 30 \text { minutes est d'obtenir une série de mots clefs } \\
\text { qui dessinent le personnage et en donnent le contour. } \\
\text { Conclusion et consignes : Réfléchir à la manière de présenter leur personnage. }\end{array}$ \\
\hline Deuxième heure & $\begin{array}{l}\text { Croquis du personnage / héros terminé sur des photocopies A4 des coupes } \\
\text { histologiques, essais de couleurs. } \\
\text { Texte : les élèves doivent écrire 4 phrases en anglais / 4 phrases en français / 1 } \\
\text { à } 4 \text { phrases en arabe (aspects scientifiques / caractéristiques de l'échantillon). }\end{array}$ \\
\hline $\begin{array}{l}\text { Troisième et qua- } \\
\text { trième heure }\end{array}$ & $\begin{array}{l}\text { Finalisation de la production des élèves (graphisme + texte) ; } \\
\text { Faire parler les élèves sur la manière dont ils ont travaillé ensemble, les faire } \\
\text { prendre conscience des difficultés qu'ils ont rencontrées et la manière dont ils } \\
\text { les ont surmontées (15 minutes). }\end{array}$ \\
\hline
\end{tabular}

Tableau 1. Déroulement de la séance Atelier. 